\title{
Designing Spatial UI as a Solution of the Narrow FOV of Microsoft HoloLens: Prototype of Virtual Museum Guide
}

\author{
Ramy Hammady ${ }^{\mathrm{a}}$, and \\ Minhua $\mathrm{Ma}^{\mathrm{a}}$ \\ ${ }^{a}$ School of Computing and Digital Technologies \\ Staffordshire University, United Kingdom \\ ramy.hammady@research.staffs.ac.uk \\ m.ma@staffs.ac.uk
}

\begin{abstract}
Since the Augmented Reality (AR) headset 'Microsoft HoloLens' released in 2016, the academic and the industrial community witnessed an obvious transformation and changes in the perception of AR applications. Despite this breakthrough, most of the HoloLens users have explicitly reported the narrow field of view (FOV) that crops the virtual augmentation from the viewer's sight to a small window of 34 degrees (Bimber \& Bruns). This limitation can result in losing premade functions and visuals in the AR application. Therefore, this study introduced attempts to design a spatial UI representing a way around the narrow FOV that HoloLens suffers from. The UI was a crucial part of AR museum system which was evaluated by 9 experts in HCI, visual communication and museum engaging studies. Results showed a positive feedback on the usability of the system and users' experience. This method can help HoloLens developers to extend their applications' functionalities with avoiding missing content.
\end{abstract}

Keywords: Microsoft HoloLens; Field of View; User Experience; Usability; Mixed Reality; Spatial UI.

\section{Introduction}

Augmented Reality Head Mounted Displays (HMD) and smart glasses are representing a revolution after the use of mobile devices. They started progressing to overcome the limited screens that users can see the blended worlds from. However, the limitation of the hardware and the image processing of the existed AR HMDs reflected a lack of usability and caused cumbersome to users (Hsieh, Jylhä, Orso, Gamberini, \& Jacucci, 2016). Therefore, the pioneering of Microsoft HoloLens was depicted on providing the opportunity to explore environments with hands-free HMD's capability (Evans, Miller, Pena, MacAllister, \& Winer, 2017). It means the device has the mobility feature with being wired with an external device or another controller. Moreover, it provides a content registration by spatial mapping (Coppens, 2017). These distinguished functionalities offer a wide usage of potential application, which subsequently reflects on more exploration on the concept of User Experience (UX) on HoloLens applications.

The spatial 3D user interfaces are commonly used in virtual reality applications (LaViola Jr, 2008). However, in the non-see-through HMDs, users cannot see the physical surroundings (D. Bowman, Kruijff, LaViola Jr, \& Poupyrev, 2004). The privilege of using spatial interface is it not constrains by the typical $2 \mathrm{D}$ desktop on mobile screens. Moreover, it open prospects to interact freely in the open space (Billinghurst, Poupyrev, Kato, \& May, 2000). According to the spatial mapping feature 
of HoloLens, AR is perfectly suitable for creating User Interface (UI) for locationaware applications (Höllerer, Feiner, Terauchi, Rashid, \& Hallaway, 1999). Thus, the UI could be allocated as a virtual layer in between the user and the physical world in the desired location. HMDs such as Microsoft HoloLens can open prospects of innovative spatial UI with collaborative interactions. The privilege of the collaborative interactions is to involve group of users to interact on the same share UI which reflects positively on content engagement and rich shared experiences.

Interaction with the spatial UI in the see-through HMDs requires a careful consideration to some factors. Some of them relevant to the user performance such as distance to be travelled to interact, size of the potential manipulated object and the length of the user (D. Bowman et al., 2004). Also, other environmental considerations are needed to take in account such as the of existence of the obstacles, level of activities or movement in the physical environment. User characteristics also should be considered such as the cognitive measures - e.g. the ability to do interactions spatially and the physical considerations such as arm length. Finally, the system considerations - e.g. the frame rate in the real-time (D. A. Bowman \& Hodges, 1999).

In spite of the uniqueness of holographic lenses of Microsoft HoloLens, the field of view is quite narrow as Bimber and Bruns stated it is $34^{\circ}$. However, Keighrey, Flynn, Murray, and Murray (2017) stated the lens have an FOV of $30^{\circ}$ by $17.5^{\circ}$. This limitation of FOV was not satisfactory of HoloLens developers and users (Bright, 2015) as it makes the AR experiences not so immersive. Others claimed it is not suitable for the user's peripheral vision (Hockett \& Ingleby, 2016). Also this constrained FOV conflicts with Milgram's definition about the adequacy of see-through displays should be achieved in its FOV (Milgram, Takemura, Utsumi, \& Kishino, 1994).

\subsection{Related Work}

Not many developers have focussed on the development of HoloLens applications yet as it is an emerging piece of technology and due to the expensiveness of its price. However, there are some novel applications that exploited HoloLens in different sectors. For instance, 'HoloMuse' that engage users with archaeological artefacts through gesture-based interactions (Pollalis, Fahnbulleh, Tynes, \& Shaer, 2017). Another research utilised HoloLens to provide in-situ assistant for users (Blattgerste, Strenge, Renner, Pfeiffer, \& Essig, 2017). HoloLens also used to provide magnification for low vision users by complementary finger-worn camera alongside with the HMD (Stearns, DeSouza, Yin, Findlater, \& Froehlich, 2017). Even in the medical applications, HoloLens contributed in 3D visualisation purposes using AR techniques (Syed, Zakaria, \& Lozanoff, 2017) and provide optimised measurements in medical surgeries(Pratt et al., 2018) (Adabi et al., 2017). Application of HoloLens extended to visualise prototype designs (DeLaOsa, 2017) and showed its potential in gaming industry (Volpe, 2015) (Alvarez, 2015) and engaging cultural visitors with gaming activities (Raptis, Fidas, \& Avouris, 2017).

The literature around UI in optical-see-through HMDs was not discussed frequently. Furthermore, the limitation of the FOV of HoloLens causes challenges in developing the UI and UX for ' $h B I M$ ' project, which leads to a rapid disappearance of the content from the user frustum view (Fonnet, Alves, Sousa, Guevara, \& Magalhães, 2017). Also in 'Holo3DGIS', the authors stated that their system cannot show the content in the 
user's visual space (Wang, Wu, Chen, \& Chen, 2018). Due to the lack of investigating this problem which is a major concern for HoloLens developers, we were motivated to find a way around to enhance the usability and the user experience.

According to the later literature, this paper we introduce:

1- A method to redesign and restructure a spatial user interface that can find a way around the limitation of the FOV.

2- Exploring the interactions of the designed UI method towards the four categories of outside factors that determined by Bowman's factors (D. A. Bowman \& Hodges, 1999) which are user performance, environmental consideration, user characteristics and system consideration. This exploration will be measure by experimental evaluation.

Keeping in mind the main aim is to increase the usefulness and immersiveness of the AR application overcoming the hardware limitation.

\section{System overview}

In order to manifest our method, it is prior to build a practical prototype and this prototype was constructed for cultural heritage guidance. The application is meant to be developed is a simple, interactive and informative system to guide visitors in museums. This system required from the user to wear Microsoft HoloLens and to explore the virtual content through spatial user interface. These interactions should be occurred with the existence of an exhibited antique in order to superimposed the content of the system as a complementary guidance.

\subsection{Functionality}

Our goal is construct the most usable UI that is capable to achieve the simplest interactions needed. The user has to interact with a spatialized 3D models, text, images, videos and buttons. The user has to deal with:

1- Doing air-tap or hand gesture on the floating virtual objects such as the virtual replica of the authentic. The user can explore the virtual item in $360^{\circ}$ so they can rotate it by dragging to right and left to spit it. The antique should be remains on its position.

2- Interacting with buttons of documentary images and scripts when needed by the visitor.

3- Interacting with a virtual guide represent a character from the same context explaining the exhibited item as a real-time virtual narrator. Also the user has the ability to click on a set of buttons to play/pause/replay to control the narrations' flow.

4- Interacting with small circles working as trigger buttons to reveal information in particular spots in the virtual replica. User can click on it by moving his head straightforward by the gaze point then doing the air-tap. 


\subsection{System architecture}

The system developments always be constructed by creating $2 \mathrm{D}$ and $3 \mathrm{D}$ assets.

Development of the system took three stages as depicted in Fig 1:

1-2D and 3D assets creation: In order to design a tempting and futuristic spatial UI design, 2D software adopted such as adobe Photoshop and Illustrator. All graphical items were designed and exported as PNG and JPG format which is going to be imported later as texture assets. Then, the $3 \mathrm{D}$ assets come to the process by creating the 3D model of the virtual guide using zBrush and Autodesk Maya. After modelling, the 3D designs required high textured materials so Substance Painter was adopted to accomplish this mission. Acquiring the virtual replica of the authentic piece went through using 3D scanners such as 3D sense and the mobile application '123D Catch'. Then, 3D scanned replica files refined and re-edited by Recap $360^{\circ}$. All 3D files are exported as FBX in order to be imported to Unity3D.

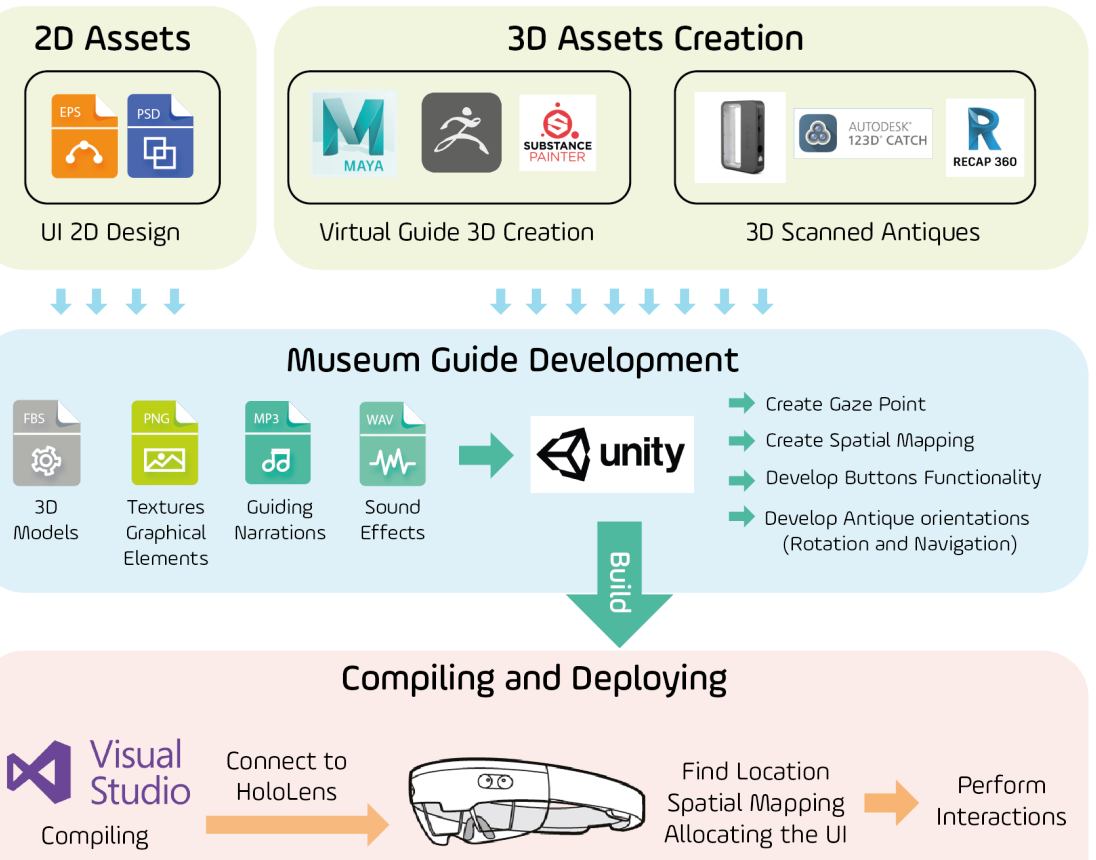

Fig. 1. System Architecture

2- Development Phase: All FBX will be imported to Unity3D project accompanied with PNG and JPG files that is considered as the main elements of the UI design. Add to it all; narrations audio files and sound effects libraries. In unity3D, project is created and the UI arranged in the scene and a set of steps are required to develop the system. Firstly, creating the gaze point in order to help the user to aim to the button that needed to be triggered. It usually appears straight ahead at the centre of the user's vision box. Secondly, the spatial mapping is developed to scan the actual 
location in order to reallocate the designed UI and the virtual guide location next to the actual antique. It is designed to respond to the user's hand gesture wherever interact. Thirdly, adding functionality to the spatial buttons such as images, scripts and controlling the narration flow. Fourthly, applying the navigation and orientation of the virtual replica in order to be controlled and responded by the user.

3- Compiling and Deploying: After building the application from Unity3D, Visual Studio's role is to compile it and deploy it to Microsoft HoloLens through a wired connection to it. Then the headset will be ready for being used. Once the user opens the application, he/she can perform the interaction - hand gesture- to allocate the spatial visuals in the desired location and the virtual guide starts at once.

\subsection{UI Design Process}

The first idea to design the spatial UI design is to be designed as a half curve and all visuals are surrounding around the user. This approach was adopted in order to make all interactive points more reachable to the user and to ease the way of interactions. As depicted in Fig 2, the brightened area is what actually the user can see from the whole scene and the semi-blacked area represents the unseen parts of the scene. In reality the blacked area is representing the actual environment without virtual content but the figure is to manifest the problem of missing content due to the narrow FOV.

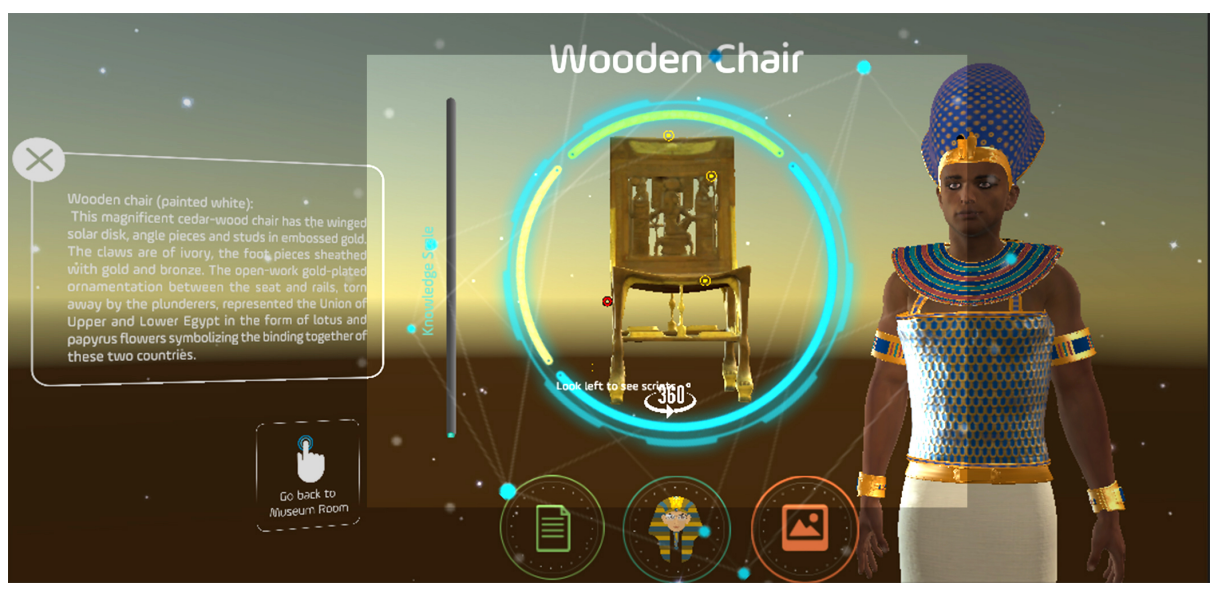

Fig. 2. Spatial UI Design as seen from HoloLens

Unsurprisingly, and due to the limited FOV, what was seen from the HoloLens view port was clipped scenes as depicted in Fig 3. Because of this uncoherent UI scene, users can not notice the existence of the missing content whether it is in the left or the right side of the user. Therefore, a series of experimental methods are conducted based on D. A. Bowman and Hodges (1999) outside factors which was part of their study. 

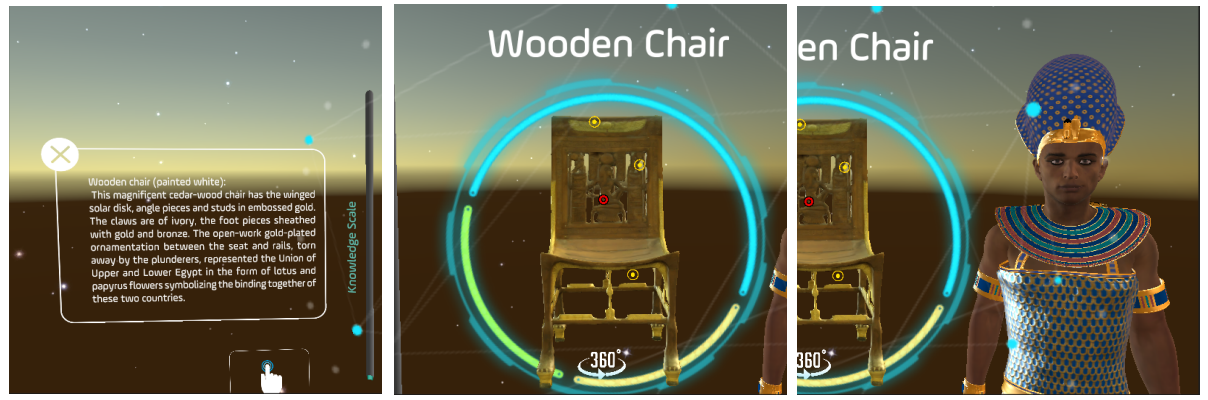

Fig. 3. Cropped scene as the HoloLens user can see

However, there are some other factors were found out aside with previous factors to accomplish the targeted goal. By considering all these factors that integrated with testing interventions, an obvious learning curve was noticed and we found a solution to enhance the usability and interactivity. Depicted in Fig. 5, the UX principles diagram required for HoloLens UI design.

1- Task characteristics: as described by (D. A. Bowman \& Hodges, 1999), they represent all aspects that influence the performance. In our UI prototype, the user has several activities that affect on the way of performing towards it. User has to walk to the UI, aim with his/her head and centre the gaze point then do air-taps. In addition to look around and watch people walking while observing the authentic exhibited item. Moreover, the interactions with the virtual replica requires a dragged hand-gesture to rotate the object. These set of activities require specific attributes to be taken care of:

a- Distance to be travelled: due to the limited FOV, what the user can see is merely quarter of the scene as mentioned before. Moreover, the user has to see the exhibited antique with the UI together. Also, it was calculated the best distance has to be from the UI is 1 meter in order to perform a proper hand interaction with the UI. However, in order to see the entire desired scene, the user has to move back 2.2 meters to see it as depicted in Fig 4. The challenge was in the scene triggers that supposed to be so close to the exhibited antique. At the first attempt, we allocated the scene triggers 1 meter away from the exhibited item. Unsurprisingly, this attempt results unrecognising the entire scene by the users however, we added a voice command or instructions to look left and right. The second attempt went better than before as we allocated the scene triggers 2.2 meters away from the exhibited item. However, the UI was so far and the location of the triggers were not in the desired location but users could see the whole scene. With minor voice and visual instructions, users came closer to interact with the UI and could realize the existence of the whole scene.

$b$ - Size of the object being manipulated: Based on our measurements in the intervened test of our participants, the best distance to do the interaction is 1 meter. Moreover, the most appropriate size that our participants felt convenient with is to be over $50 \mathrm{~cm}$ height and $50 \mathrm{~cm}$ width. It is worthy to mention that 
most of the participants were exposed to minimal instructions on how to do the air-tap and make the dragging gesture.

2- Environmental characteristics: The environment of using and interacting with HoloLens requires an adequate space. Therefore, we consider the characteristic of the space by involving some variables in the process of interactions.

a- Number of obstacles: While running the application, it was concluded that the user requires an empty area in front of him/her to place the visuals. If some people pass in front of the user, it might change the location of the UI and that's due to the deforming of the spatial mapping of the actual location. So, this problem is commonly in museums, it is expected to have many visitors at the same spot.

b- Lighting levels: It is preferable to display visuals in low levels of lighting conditions and the visuals opacity increases with interior lighting conditions. Sun lights decreases the opacity percentages and the visuals start to lose its opaqueness.
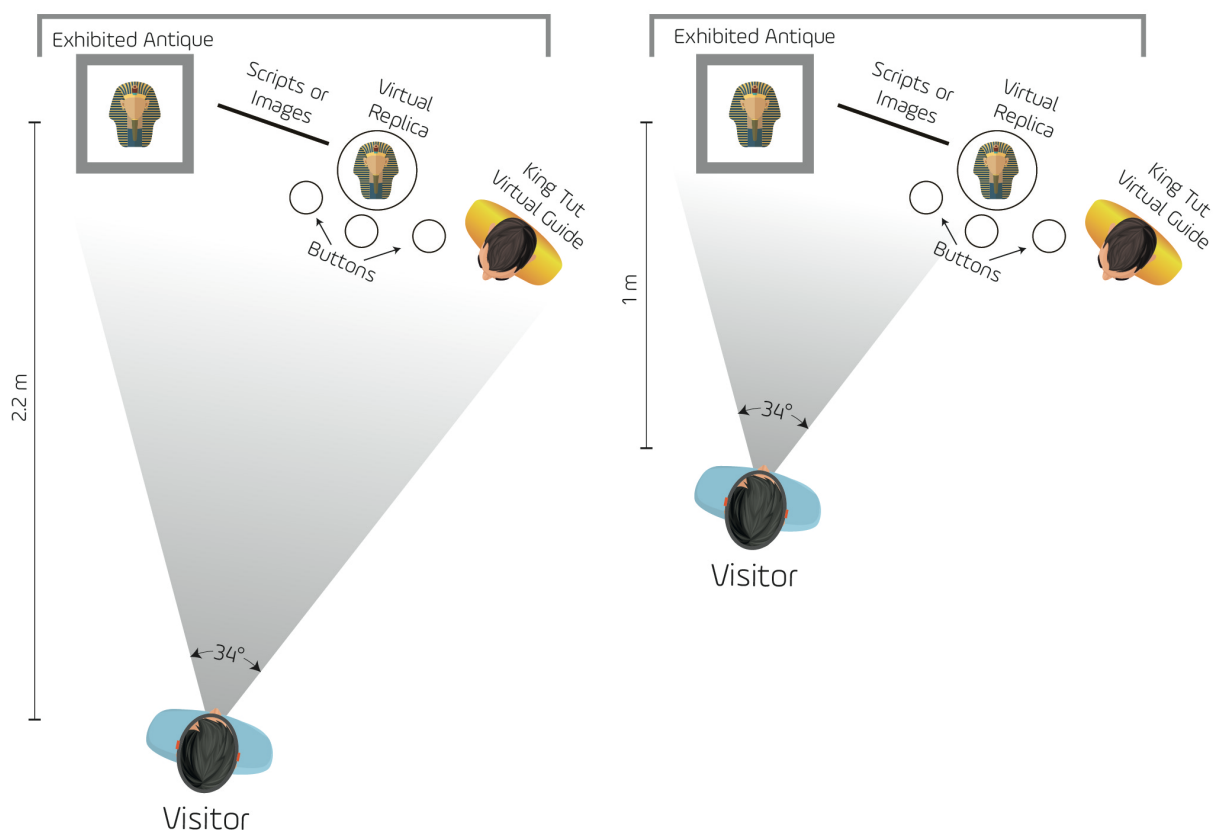

Fig. 4. Visualising the entire seen from different locations

3- User characteristics: All aspects related to the user himself/herself regarding the physical and cognitive attributes.

a- Cognitive measures: Participated group were instructed minimally the way the interact and do the air-tap. During the experimental phases, we noticed different levels of acquiring the interaction skills, which reflects an uneven retention of 
the instructions. Getting used of the HoloLens interactions takes time with some people and no time with others.

b- Physical aspects: The diversity of the participants' heights were noticed during testing interventions. We designed the visuals are appropriate to 1.70 meter for the person's height. However, we noticed that shorter participants tend to look up to the visuals, which is a cumbersome for them and cause pain in their necks after time. Likewise, the participant who their height is longer than $1.70 \mathrm{~cm}$. After several attempts, we were driven to scale the whole UI based on the person's height. Once the scene opened, it calculates the distance between the ground and the camera of the HoloLens. Then, it scales the whole UI based on it. Eventually, it made the participants feel more convenient.

4- System characteristics: All aspects that relevant to the headset or the application developed or the hardware specifications.

a- Frame rate of the scene: It was noticed a rapid streaming of the physical visualisation that combined with the virtual content when the complexity of 3D graphics is in minor levels. On contrary, if the current frame that the user is observing from the HoloLens viewport has loads of complex 3D models, the frame rate will come to 15 - 20 frame per second. It also could cause lags and delay of rendering the current frame. So, from the spatial design perspective, it is recommended to distribute the complex $3 \mathrm{D}$ visuals around the space with adequate room in order to avoid seeing them together in one frame.

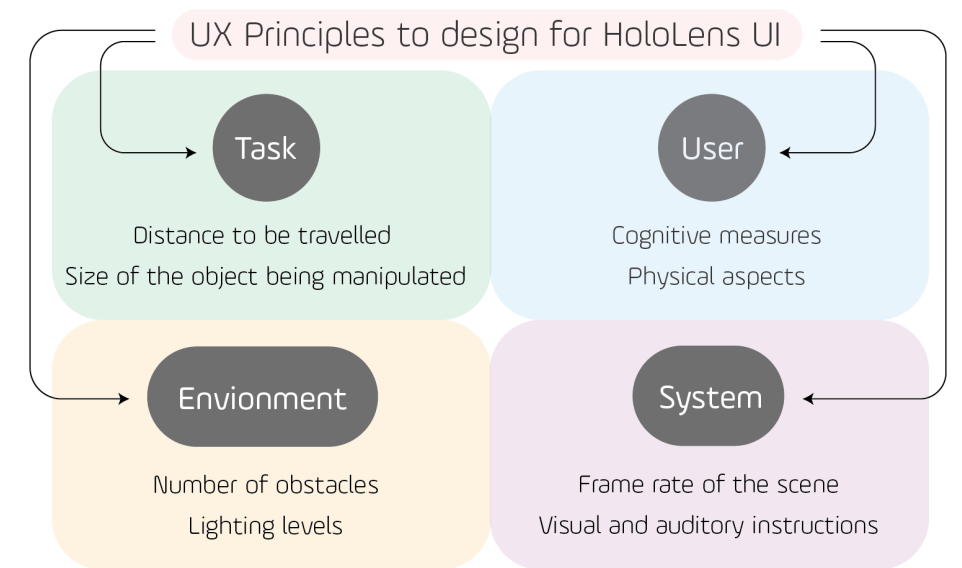

Fig. 5. UX principles for HoloLens UI Design developed from (D. A. Bowman \& Hodges, 1999).

$b$ - Visual and auditory instructions: From the UX concepts, the user should be aware of the all visuals that designed to be seen or to be heard. To loose recognising or seeing a visual content from the user cause a lack of user experience. Therefore, we used a 'tag along method', which gives the user a visual clue that points to the location of the virtual content in the space around the object. This method adopted by Fonnet et al. (2017). It ensures that the 
content is constantly a glace away from the users. We also used auditory instructions to compensate the visual instructions if there is no room for the later method.

\section{Prototype Evaluation}

We opted for a field study demanding the involvement and participation of actual users. The aim of the evaluation is to investigate the usability aspect and the other unexplored aspects that might occur during the system usage. The evaluation's nature was a completed simulation of the actual system that could be applied practically in museums.

\subsection{Method}

we conducted an evaluation in the university library where we invited 9 participants and they are experts in different disciplines such as in Human Computer Interactions (HCI), visual communication and museum engaging studies. The research employed experts to do a discrete evaluation on aspects some of them were common to the participant evaluation and other relevant to their expertise. Worthy to mention that some similar studies employed disciplines' experts to ensure the validity to the evaluation process (Karoulis, Sylaiou, \& White, 2006). They were instructed prior the experiment by a brief tutorial on the way of interaction as depicted in figure 6 . The evaluation method adopted the quantitative method as the research instrument was semi-structured questionnaires that include open questions. These questionnaires were piloted prior the experiment through other experts to make sure the questions are clear and answer the research objectives The quantitative method comprised of two types: textual analysis and numeric analysis. The textual questions were designed based on 1 to 5 Likert scale. The experiment took from 5-10 min per participant. In table 1 , the demographics of participant is manifested based on their age group, expertise area and years of experience.

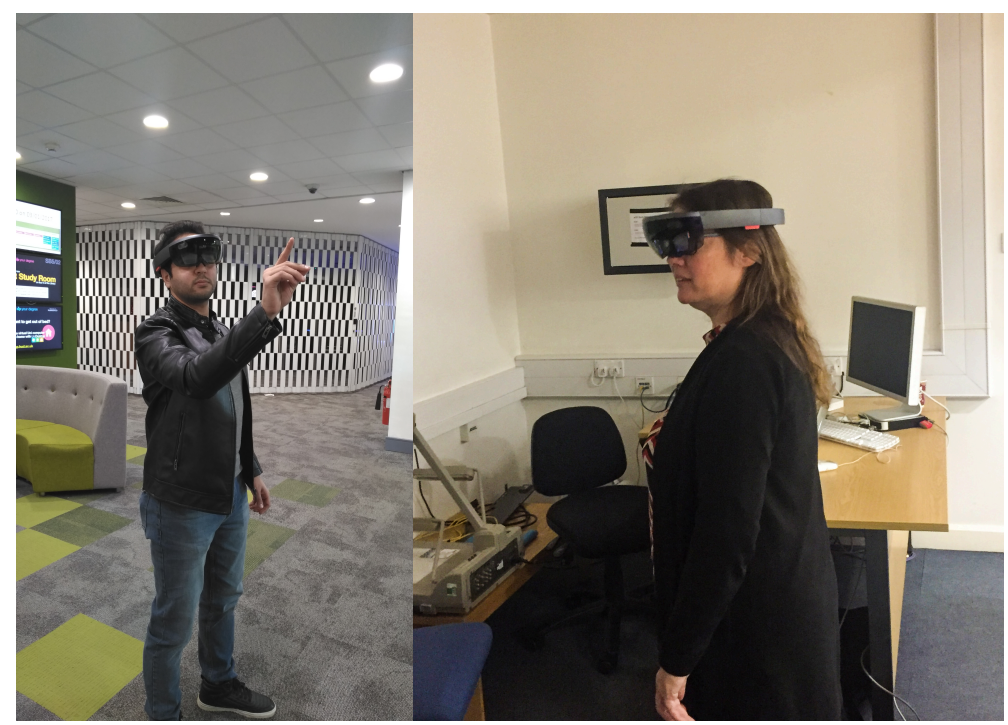

Fig. 6. Participants testing the system 


\subsection{Results}

The numeric data in table 2 represents the measurements of the usability aspect including the experience of interactions and preforming the functions desired. The first question was the lowest positive percentage of respondents were about the convenience of the headset and if it is comfortable during the usage of the prototype or not. As participants stated "Slightly heavy perhaps" and another said "It was a bit heavy on my neck, so I would not want it on too much". It is worthy to mention that the headset is $579 \mathrm{~g}$ (Microsoft, 2015). The following question wonders if it causes dizziness, or headache, however the responds were highly positive. A participant commented "The HoloLens was much better than VR headsets, there was no disorientation or loss of the horizon, I was immersed in the location without losing track of my surroundings. Good experience". The $3^{\text {rd }}$ question's responses also were quite positive, however a participant "I was glad to be reminded to look up and down". The $4^{\text {th }}$ question was regarding the air-tap and the comments were quite convincing. A participant stated "I had to learn how to do this - but that was part of the fun" and another commented "Yes, after minimal guidance". The $5^{\text {th }}$ question was examining the core of our theoretical study and the comments were progressive as a participant commented "Much more interactive than anticipated-love that you can more around the scene and look in all directions". However, another participant stated "It is required some time to deal with $i t^{\prime \prime}$. The $6^{\text {th }}$ question also examines the main theoretical contribution and the percentage was similar to the previous question. Participants responses regarding this question were all positive as someone stated "This became easier the move I used the device" and another commented "Generally, very easy to use".

Table 1. Participants demographics

\begin{tabular}{|c|c|c|c|}
\hline Discipline expertise & $\begin{array}{l}\text { Male/ } \\
\text { Female }\end{array}$ & $\begin{array}{l}\text { Years of } \\
\text { Experience }\end{array}$ & Age Group \\
\hline $\begin{array}{l}\text { Academic and professional expert in } \\
\text { Visual communication and Arts }\end{array}$ & $\mathrm{F}$ & 22 & $45-60$ \\
\hline $\begin{array}{l}\text { Expert at public engagement in } \\
\text { museums }\end{array}$ & $\mathrm{F}$ & 7 & $31-45$ \\
\hline Expert in museum curatorship & M & 7 & $25-30$ \\
\hline Expert in museum curatorship & $\mathrm{F}$ & 6 & $31-45$ \\
\hline Expert in museum curatorship & $\mathrm{F}$ & 4 & $25-30$ \\
\hline Expert in $\mathrm{HCI}$ and visual interactions & $\mathrm{F}$ & 9 & $31-45$ \\
\hline $\begin{array}{l}\text { Data manager and responsible for } \\
\text { enhancing the museum visitor } \\
\text { engagement }\end{array}$ & $\mathrm{F}$ & 2 & $31-45$ \\
\hline Expert in museum curatorship & M & 10 & $31-45$ \\
\hline $\begin{array}{l}\text { Academic and professional expert in } \\
\text { museum curatorship }\end{array}$ & $\mathrm{M}$ & 8 & $31-45$ \\
\hline
\end{tabular}


Table 2. Usability Aspect

$(1=$ Strongly disagree to $5=$ Strongly agree. $\mathrm{N}=9)$

\begin{tabular}{|c|c|c|c|c|c|c|c|}
\hline Measure & $\begin{array}{l}\text { Strongly } \\
\text { agree }\end{array}$ & Agree & Neutral & Disagree & $\begin{array}{l}\text { Strongly } \\
\text { disagree }\end{array}$ & Mean & $\begin{array}{l}\text { Std. } \\
\text { dev. }\end{array}$ \\
\hline $\begin{array}{l}\text { I found the headset } \\
\text { comfortable to } \\
\text { wear }\end{array}$ & $0.0 \%$ & $77.8 \%$ & $11.1 \%$ & $4.3 \%$ & $0.0 \%$ & 3.67 & .707 \\
\hline $\begin{array}{l}\text { I did not } \\
\text { experience nausea, } \\
\text { dizziness, or } \\
\text { headache }\end{array}$ & $77.8 \%$ & $22.2 \%$ & $0.0 \%$ & $0.0 \%$ & $0.0 \%$ & 4.78 & .441 \\
\hline $\begin{array}{l}\text { I could look } \\
\text { around the room } \\
\text { comfortably }\end{array}$ & $10.6 \%$ & $44.7 \%$ & $38.3 \%$ & $6.4 \%$ & $0.0 \%$ & 4.44 & 1.014 \\
\hline $\begin{array}{l}\text { I could do air tap } \\
\text { on the virtual } \\
\text { objects } \\
\text { appropriately }\end{array}$ & $33.3 \%$ & $55.6 \%$ & $11.1 \%$ & $0.0 \%$ & $0.0 \%$ & 4.22 & .667 \\
\hline $\begin{array}{l}\text { I could interact } \\
\text { with the user } \\
\text { interface as I } \\
\text { expected }\end{array}$ & $44.4 \%$ & $33.3 \%$ & $11.1 \%$ & $11.1 \%$ & $0.0 \%$ & 4.11 & 1.054 \\
\hline $\begin{array}{l}\text { I could do all } \\
\text { functions I desired }\end{array}$ & $33.3 \%$ & $55.6 \%$ & $55.6 \%$ & $11.1 \%$ & $0.0 \%$ & 4.11 & .928 \\
\hline
\end{tabular}

\subsection{Discussion and Conclusion}

This research contributes theoretically by forming the UX principals for HoloLens UI applications as way to overcome the UI problems that stated in the literature. Our experiment was driven due to the formed UX principles in, which can enhance the usability aspect which was affected by the narrow FOV of HoloLens that was stated clearly in the literature. The conducted experiment introduced an empirical evidence through a HoloLens system prototype, which provides strong support for our hypothesis. Hence, this prototype was developed according to the UX principles in order to measure the ability to overcome the FOV UI problem.

Also this paper manifests the system structure with the pipeline of the designed prototype that can be used to guide visitors in museums. This system is featured to have a personalised virtual guide, intractable virtual replica and holographic UI presented aside with the exhibited antique. According to the positive results, the prototype proved its usability and accessibility to a group of experts with different expertise that relevant to the prototype cross-disciplinary nature. The theoretical contribution is quite helping the UX designers and developers to overcome the mentioned problem if they can consider these principles during their pipeline and testing phases. Also the techniques are flexible to be applied to any application has the spatial UI design and intractable 
floating buttons. The UX model also consider users who has different heights and also consider different environment natures.

Despite the HoloLens enabled us to develop prototypes with genuine AR applications, it still has limitations that should be considered in the future versions of it. For instance, the unstable spatial mapping function was witnessed when someone crossed in front of the user. Also the device is bulky for some users, so they could not able to wear it for long periods which means it is not so much acceptable in museum long tours. Moreover, the real-time rendered frame should not have so much graphics $-3 \mathrm{D}$ models and textures-, otherwise it could cause lags and delays of real-time rendering.

Enhancing the museum experience through immersive technologies reflects explicitly on the tourism industry. It absolutely encourages visitors to come to museums to experience the technological tool and to enjoy it. Hence, museum managements need to invest time and resources on these vital tools as it might be the next revolutionary gadget in museums that can reshape the mental image of museums.

\subsection{Future work}

We seek for adding more usability and enriching the user experience to the system so that we are aiming to add voice recognition and text-to-speech techniques. We still not sure if the voice recognition can practically work with some languages e.g. Arabic language. Also we envision a futuristic AR system that can present visuals beyond the spatial UI and can depict holograms everywhere around the user. That system can consider the user is in the centre of a sphere of interactive worlds. We also aim to include games at specific zones of museums to enrich the level of interactivity and empower the content with contextual interesting stories. These games should have some motivational actions such as exploring and investigation the rooms to discover clues for rewards and reaching milestones. These actions can encourage the physical interactions within the museum context. Hopefully, this expanded system could achieve more aims with newly developed HoloLens or headset with widen FOV.

\section{Acknowledgements}

We are very thankful to Polina Zioga who helped in designing the questionnaire and ethics forms and also grateful to all experts who participated in the evaluation process.

\section{References}

Adabi, K., Rudy, H., Stern, C. S., Weichman, K., Tepper, O., \& Garfein, E. S. (2017). Optimizing Measurements in Plastic Surgery through Holograms with Microsoft Hololens. Plastic and Reconstructive Surgery Global Open, 5(9 Suppl).

Alvarez, E. (2015). Microsoft shows off 'Minecraft' built specifically for HoloLens. Retrieved from https://www.engadget.com/2015/06/15/microsoft-minecrafthololens/

Billinghurst, M., Poupyrev, I., Kato, H., \& May, R. (2000). Mixing realities in shared space: An augmented reality interface for collaborative computing. Paper presented at the Multimedia and Expo, 2000. ICME 2000. 2000 IEEE International Conference on. 
Bimber, O., \& Bruns, E. (2011). PhoneGuide: Adaptive Image Classification for Mobile Museum Guidance.

Blattgerste, J., Strenge, B., Renner, P., Pfeiffer, T., \& Essig, K. (2017). Comparing conventional and augmented reality instructions for manual assembly tasks. Paper presented at the Proceedings of the 10th International Conference on PErvasive Technologies Related to Assistive Environments.

Bowman, D., Kruijff, E., LaViola Jr, J. J., \& Poupyrev, I. P. (2004). 3D User interfaces: theory and practice, CourseSmart eTextbook: Addison-Wesley.

Bowman, D. A., \& Hodges, L. F. (1999). Formalizing the design, evaluation, and application of interaction techniques for immersive virtual environments. Journal of Visual Languages \& Computing, 10(1), 37-53.

Bright, P. (2015). HoloLens: Still magical, but with the ugly taint of reality. Retrieved from https://arstechnica.com/gadgets/2015/05/hololens-still-magical-but-withthe-ugly-taint-of-reality/

Coppens, A. (2017). Merging real and virtual worlds: An analysis of the state of the art and practical evaluation of Microsoft Hololens. arXiv preprint arXiv:1706.08096.

DeLaOsa, J. (2017). Ford Designs Next-Gen Cars Using Microsoft's HoloLens. Retrieved from https://www.ecnmag.com/blog/2017/09/ford-designs-next-gencars-using-microsofts-hololens

Evans, G., Miller, J., Pena, M. I., MacAllister, A., \& Winer, E. (2017). Evaluating the Microsoft HoloLens through an augmented reality assembly application. Paper presented at the Degraded Environments: Sensing, Processing, and Display 2017.

Fonnet, A., Alves, N., Sousa, N., Guevara, M., \& Magalhães, L. (2017). Heritage BIM integration with mixed reality for building preventive maintenance. Paper presented at the Computação Gráfica e Interação (EPCGI), $201724^{\circ}$ Encontro Português de.

Hockett, P., \& Ingleby, T. (2016). Augmented reality with HoloLens: Experiential architectures embedded in the real world. arXiv preprint arXiv:1610.04281.

Höllerer, T., Feiner, S., Terauchi, T., Rashid, G., \& Hallaway, D. (1999). Exploring MARS: developing indoor and outdoor user interfaces to a mobile augmented reality system. Computers \& Graphics, 23(6), 779-785.

Hsieh, Y.-T., Jylhä, A., Orso, V., Gamberini, L., \& Jacucci, G. (2016). Designing a willing-to-use-in-public hand gestural interaction technique for smart glasses. Paper presented at the Proceedings of the 2016 CHI Conference on Human Factors in Computing Systems.

Karoulis, A., Sylaiou, S., \& White, M. (2006). Usability evaluation of a virtual museum interface. Informatica, 17(3), 363-380.

Keighrey, C., Flynn, R., Murray, S., \& Murray, N. (2017). A QoE evaluation of immersive augmented and virtual reality speech \& language assessment applications. Paper presented at the Quality of Multimedia Experience (QoMEX), 2017 Ninth International Conference on.

LaViola Jr, J. J. (2008). Bringing VR and spatial 3D interaction to the masses through video games. IEEE Computer Graphics and Applications, 28(5).

Microsoft. (2015). HoloLens hardware details. Retrieved from https://developer.microsoft.com/en-us/windows/mixedreality/hololens hardware details 
Milgram, P., Takemura, H., Utsumi, A., \& Kishino, F. (1994). Augmented Reality: A class of displays on the reality-virtuality continuum. Telemanipulator and Telepresence Technologies, 2351.

Pollalis, C., Fahnbulleh, W., Tynes, J., \& Shaer, O. (2017). HoloMuse: Enhancing engagement with archaeological artifacts through gesture-based interaction with holograms. Paper presented at the Proceedings of the Tenth International Conference on Tangible, Embedded, and Embodied Interaction.

Pratt, P., Ives, M., Lawton, G., Simmons, J., Radev, N., Spyropoulou, L., \& Amiras, D. (2018). Through the HoloLens ${ }^{\mathrm{TM}}$ looking glass: augmented reality for extremity reconstruction surgery using $3 \mathrm{D}$ vascular models with perforating vessels. European Radiology Experimental, 2(1), 2.

Raptis, G. E., Fidas, C., \& Avouris, N. (2017). Cultural Heritage Gaming: Effects of Human Cognitive Styles on Players' Performance and Visual Behavior. Paper presented at the Adjunct Publication of the 25th Conference on User Modeling, Adaptation and Personalization.

Stearns, L., DeSouza, V., Yin, J., Findlater, L., \& Froehlich, J. E. (2017). Augmented Reality Magnification for Low Vision Users with the Microsoft Hololens and a Finger-Worn Camera. Paper presented at the Proceedings of the 19th International ACM SIGACCESS Conference on Computers and Accessibility.

Syed, A., Zakaria, A., \& Lozanoff, S. (2017). dark Room To Augmented Reality: Application Of Hololens Technology For Oral Radiological Diagnosis. Oral surgery, oral medicine, oral pathology and oral radiology, 124(1), e33.

Volpe, J. (2015). Disney Infinity might make its way to Microsoft's HoloLens. Retrieved from https:/www.engadget.com/2015/07/01/disney-infinity-mightmake-its-way-to-microsoft-hololens/

Wang, W., Wu, X., Chen, G., \& Chen, Z. (2018). Holo3DGIS: Leveraging Microsoft HoloLens in 3D Geographic Information. ISPRS International Journal of GeoInformation, 7(2), 60 . 